\title{
Effect of loss on the topological features of dimer chain described by the extended Aubry-André-Harper model
}

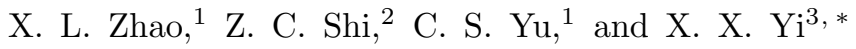 \\ ${ }^{1}$ School of Physics and Optoelectronic Technology, \\ Dalian University of Technology, Dalian 116024, China \\ ${ }^{2}$ Department of Physics, Fuzhou University, Fuzhou 350002, China \\ ${ }^{3}$ Center for Quantum Sciences and School of Physics, \\ Northeast Normal University, Changchun 130024, China
}

(Dated: October 5, 2018)

\begin{abstract}
By introducing loss to one sublattice of a dimer chain described by the extended Aubry-André or Harper (AAH) model, we study the topological features including the edge states, spectrum and winding number of the chain. We find that the parameter region for the system to have real band-gap-closing is increased due to the loss, and the average displacement of the single excitation can still witness the topological features of the chain in the presence of loss. The robustness of the zero energy eigenstate against four kinds of disorders is also examined. A feasible experiment setup based on coupled waveguides to observe the prediction of this paper is proposed.
\end{abstract}

\section{INTRODUCTION}

In 1980, Aubry and André showed the existence of localization phase transition through a $1 \mathrm{D}$ tight binding quasiperiodic system [1] described by the one dimensional (1D) lattice model (called Aubry and André model now). The model can be mapped into the $2 \mathrm{D}$ rectangular lattice for integer quantum Hall effect 2, 3, by using Landau gauge for the magnetic field, where the periodic character is determined by the flux quanta penetrating each rectangle lattice. Then the terminology 'AAH model' is used widely to abbreviate the Aubry-André and Harper models. The period for the $1 \mathrm{D}$ lattice is usually a trigonometrical function of length and it can be turned flexibly in principle. But to investigate intriguing properties, the extreme huge magnetic density is a bottleneck currently in experiments for solid systems [3 5].

With the development of topological materials, the AAH model has been explored in the view of topological aspects 6 10, which can bridge the quantum Hall effect (QHE) [11-14] and the topological insulator [15]. For example the 1D AAH model has the topologically protected edge states corresponding to the gapless edge states in QHE. Recent experiments [7, 16] have realized the quasiperiodic AAH model in optical lattices and the signature of a localization transition [16] was observed in agreement with the theory [1].

The array of waveguides is a valid platform to explore topological insulators due to the developed manufacturing and designing technique [7, 18]. However, the loss represented by non-Hermitian terms in the system is usually inevitable in practice [17, 18. It is then reasonable to consider the influence of loss to the topological properties. Considering that the AAH model may be implemented in such optical systems, we employ in this work a dimer(two sites in each cell) AAH model with loss on

* E-mail: yixx@nenu.edu.cn one of the sites in each cell, to explore the topological properties in terms of the hopping and on-site modulation phases.

Disorders exist widely in practical systems. In the original work by Aubry and André [1], the incommensurate potentials mimic the disorders leading to localization transition. Although extensive theoretical works for the effects of disorders in the AAH model have been done 19 23 , the study of robustness of topological states against these disorders is lacking. It is well known that the degenerate zero energy edge states are the edge states. Then we will explore the robustness of zero energy edge states against the disorders on the chain. Four kinds of disorders are considered: intra-cell hopping disorders, inter-cell hopping disorders, on-site disorders and nonHermitian disorders. We find that the zero energy edge states are robust against the disorders in an interval of strength except for the on-site disorders since on-site disorders destroy the chiral symmetry. We also find that the non-Hermitian disorders 'draw' the real energy band towards zero energy.

We will explore the aforementioned issues by considerations that both the hopping amplitude and on-site potentials are modulated in the real space commensurate with the lattice in the extended AAH model [8, 24]. The setups for the realization of this model is feasible in coupled waveguides with modulated lattice spacings and lattice widths, details of which are presented latter on.

This work is organized as follows. In section [II, we introduce a dimer chain described by the extended $\mathrm{AAH}$ model. In terms of hopping modulation phase, we study the influence of loss on one sublattice to the topological features based on the mean displacement of the single excitation on the chain. In section III] we examine the topological properties of the system in terms of on-site modulation phase. In section IV] we study the robustness of the zero energy edge mode against four kinds of disorders. In section $\mathrm{V}$, we propose an experimental setup to observe the prediction based on coupled single mode 
waveguides. Finally, we conclude in section VI.

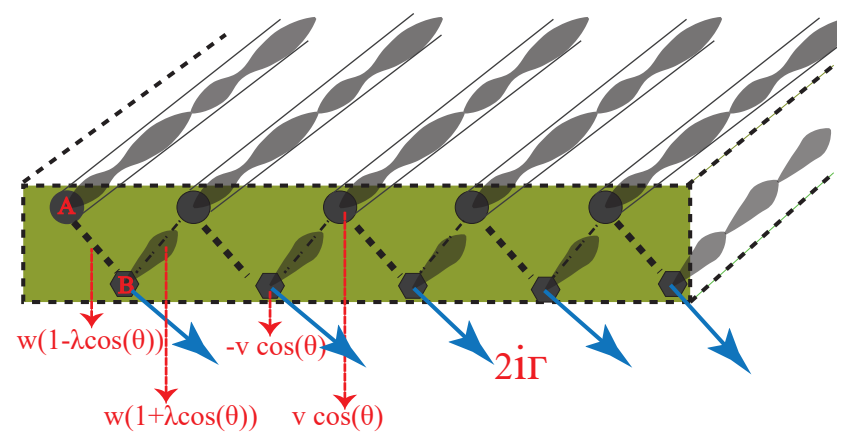

Figure 1. The sketch for the extended AAH model realized in coupled waveguides. The modulations are functions of sequence of the juxtaposed waveguides. The loss occurs on sublattice composed of B-sites. The time evolution corresponds to the propagation of light excitation along the waveguides.

\section{TOPOLOGICAL PROPERTIES IN TERMS OF HOPPING MODULATION PHASE}

Consider an extended 1D AAH model with modulated nearest-neighbor hopping interactions in real space described by the following tight-binding Hamiltonian

$$
\begin{aligned}
H= & \sum_{n=1}^{N-1} w[1+\lambda \cos (2 \pi b n+\theta)] c_{n+1}^{\dagger} c_{n}+\text { h.c. } \\
& +\sum_{n=1}^{N} v \cos \left(2 \pi b n+\theta_{v}\right) c_{n}^{\dagger} c_{n} .
\end{aligned}
$$

This 1D chain consists of $N$ sites $(n=1,2, \ldots, N)$. Here $c_{n}^{\dagger}$ and $c_{n}$ are the polarized fermionic creation and annihilation operators on site $n$. When we only consider a single excitation on the chain, the operators $c_{n}^{\dagger}$ and $c_{n}$ might describe bosons. The terms in the first line in (1) represent the kinetic energy or the nearest-neighbor hoppings. $w$ is the strength which is taken as the energy unit throughout this work and the dimensionless $\lambda$ indicates the modulation amplitude. The last terms describe the modulated on-site potentials where $v$ is the strength. $\theta$ and $\theta_{v}$ are the modulation phases.

When $b$ is an irrational number, the diagonal AAH model $(\lambda=0, v \neq 0)$ possesses a localization transition as $v$ crosses a critical value [1, 25, 26. In one-dimensional quasicrystals system, the topologically protected boundary states equivalent to the edge states in quantum Hall system appears when $b$ takes irrational values [7].

We will focus on the case that $b$ is a rational number when the hopping and on-site potential modulations have the periodicity of $1 / b$ determined by the magnetic field penetrating the $2 \mathrm{D}$ counterpart of the $1 \mathrm{D}$ chain $[2,3]$.
When $\lambda=0(v=0)$, the Hamiltonian describes the diagonal AAH model which can be derived from the $2 \mathrm{D}$ Hofstadter model 8, 24.

In this paper, we will set the modulation phases of on-site potential fulfilling the condition $\theta=\theta_{v}+\pi$. In the experiment, the setups can be designed to make $\theta$ and $\theta_{v}$ tunable independently such as in coupled optical waveguides systems since the phases is determined by modulating the spacing between the waveguides and the widths [7, 8]. Thus it is reasonable to assume $\theta_{v}=\theta+$ $\phi$ where $\phi$ is independent of $\theta$. We will focus on the topological properties of this model in terms of the two modulation phases $\theta$ and $\phi$ in the following.

To simplify the problem, we consider $b=1 / 2$ case when the odd and even sites feel different commensurate hopping and on-site potentials. Then we denote the annihilation operators for the odd sites (A-sites) as $\hat{a}$ and $\hat{b}$ for even sites (B-sites) and treat an odd-even combination of the sites on the original chain as one cell, namely, the chain of dimer. In order to investigate the influence of loss to the topological properties of this system, we introduce non-Hermitian terms to B-sites with strength $i 2 \Gamma$, see Fig.1. The Hamiltonian reads,

$$
\begin{aligned}
H= & H_{\text {odd }}+H_{\text {even }}, \\
H_{\text {odd }}= & \sum_{n: \text { odd }}[1-\lambda \cos (\theta)] b_{n}^{\dagger} a_{n} \\
& +h . c-v \cos \left(\theta_{v}\right) a_{n}^{\dagger} a_{n}, \\
H_{\text {even }}= & \sum_{n: \text { even }}[1+\lambda \cos (\theta)] a_{n+1}^{\dagger} b_{n} \\
& +h . c+\left(v \cos \left(\theta_{v}\right)-2 i \Gamma\right) b_{n}^{\dagger} b_{n} .
\end{aligned}
$$

In this dimer Hamiltonian, the hopping terms in $H_{\text {odd }}$ are intra-cell hoppings and those in $H_{\text {even }}$ are the inter-cell hoppings.

The emergence of the edge states with isolated eigenenergies in the energy gap for a system with open boundary condition is a signature of nontrivial topological properties corresponding to the nonvanishing topological number in momentum space. Considering the map from the one-dimensional version of AAH model to the twodimensional counterpart, we confirm that the modulation phase $\theta$ is treated as a momentum component hereafter. Thus the 'momentum space' is not puzzled. Next, we consider the topological properties in terms of $\phi$. And this phase is also treated as a momentum component after Fourier transformation. By transforming the real space Hamiltonian to the one in momentum space under the periodic boundary assumption, we can examine the topological phases by topological invariant.

In the basis of $\left[\begin{array}{ll}a_{k}^{\dagger} & b_{k}^{\dagger}\end{array}\right]^{T}$, the Hamiltonian in the mo- 

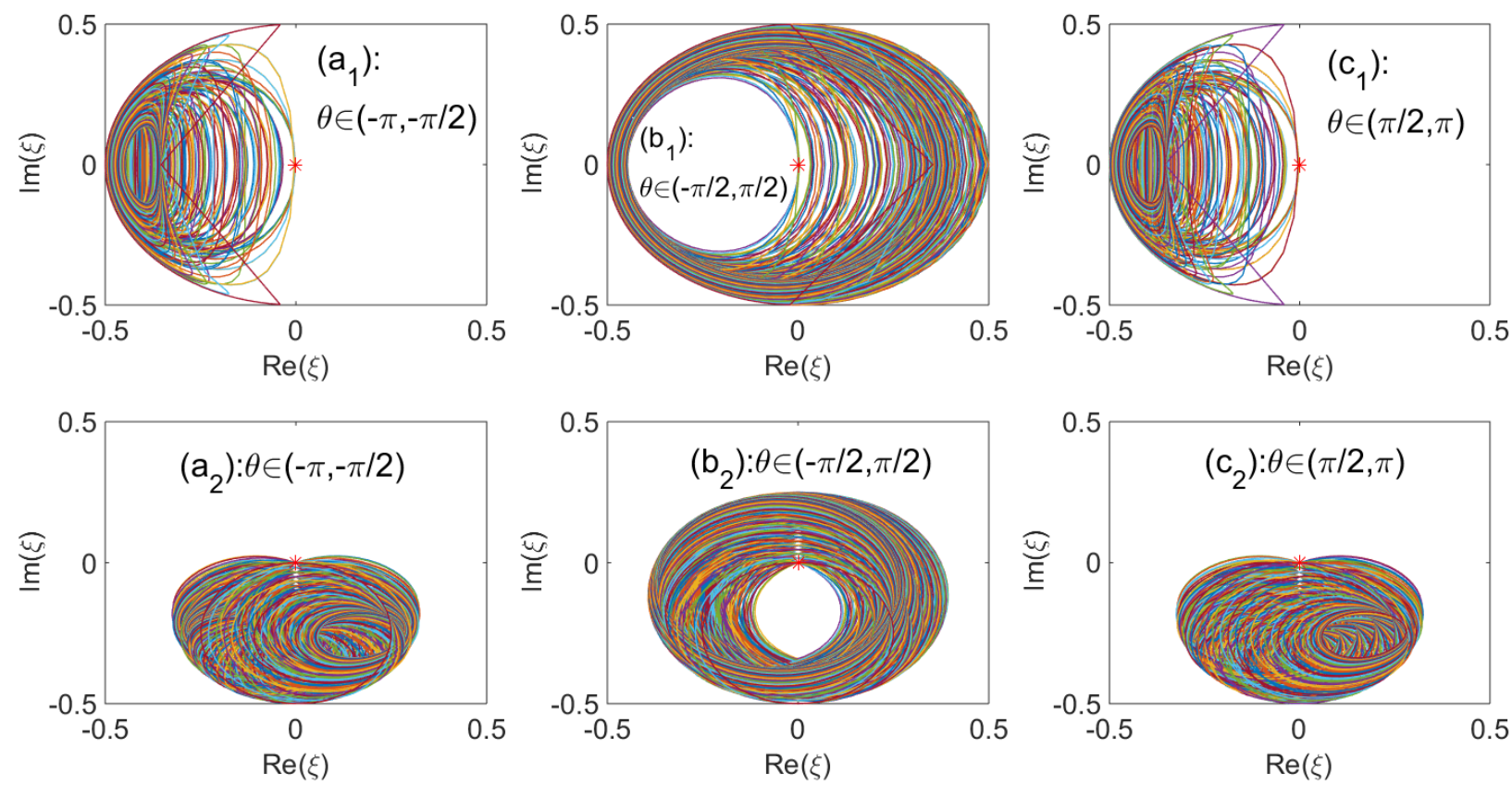

Figure 2. $\left(a_{1}\right),\left(b_{1}\right)$ and $\left(c_{1}\right)$ are the trajectories of the wind number $\xi$ in the complex plane for the Hermitian case. $\left(a_{2}\right)$, $\left(b_{2}\right)$ and $\left(c_{2}\right)$ are those in the non-Hermitian Hamiltonian case with $\Gamma=2$. The red star is the origin to indicate whether the trajectories of $\xi$ wind around it. $\phi \in[0,2 \pi]$ are shown in all these cases. The winding in this figure coincides with the emergence of edge states in Fig 3 and Fig 4

mentum space can be written as,

$$
\begin{aligned}
H_{k}= & \vec{h} \bullet \sigma=h_{x} \sigma_{x}+h_{y} \sigma_{y}+h_{z} \sigma_{z}, \\
& h_{x}=1-\lambda \cos (\theta)+[1+\lambda \cos (\theta)] \cos (k), \\
& h_{y}=[1+\lambda \cos (\theta)] \sin (k), \\
& h_{z}=-v \cos (\theta+\phi)+i \Gamma,
\end{aligned}
$$

where we have added $-i \Gamma$ to $h_{z}$ to describe the loss. The dispersion relation by $H^{2}=E^{2} \mathbf{I}$ is

$$
E_{ \pm}= \pm \sqrt{h_{x}^{2}+h_{y}^{2}+h_{z}^{2}}= \pm \sqrt{A^{2}+B^{2}+C-D},
$$

where $A^{2}=2\left(1+\lambda^{2} \cos ^{2}(\theta)\right), B^{2}=v^{2} \cos ^{2}(\theta+\phi), C=$ $2\left(1-\lambda^{2} \cos ^{2}(\theta)\right) \cos (k)$ and $D=\Gamma^{2}+i 2 v \Gamma \cos (\theta+\phi)$. One of the eigenvectors of $H_{k}$ with eigenvalue $E_{+}$is

$$
\left|u_{k,-h}\right\rangle=\left(\begin{array}{c}
\phi_{k}^{A} \\
\phi_{k}^{B}
\end{array}\right)=\left(\begin{array}{c}
\cos \eta \\
\sin \eta
\end{array}\right),
$$

where $\eta$ fulfill $\tan (2 \eta)=\left(h_{x}-i h_{y}\right) / h_{z}$. Since the gauge does not influence the topological properties, we have neglected the phase related to gauge.

Based on the mentioned above, the topological property can be expressed by the wind number which can be defined in different ways such as the ratio of the two components of one of the eigenstates in the momentum representation [17]. Additionally, the product of the two components:

$$
\xi=\phi_{k}^{A} \phi_{k}^{B}=\frac{1}{2}\left(\bar{h}_{x}-i \bar{h}_{y}\right),
$$

can also be used as a wind number in the complex plane of $\left(\bar{h}_{x}, \bar{h}_{y}\right)$ where $\bar{h}_{x}$ and $\bar{h}_{y}$ are $h_{x}$ and $h_{y}$ divided by $2 \operatorname{Re}(E)+2 i \Gamma$, where $E$ is the eigenenergy and $\operatorname{Re}(\bullet)$ returns the real part of $\bullet$. Whether the trajectory of $\xi$ wraps the origin is an signature of topological property for the system and independent of which eigenstate being used. We show the trajectory of $\xi$ in Fig. 2 for three intervals of $\theta$. To plot these figures, $\phi \in[0,2 \pi]$ have been randomly examined. It can be seen that although the trajectories of $\xi$ are different for the Hermitian and non-Hermitian cases, the topological regions are same in terms of $\theta$. Considering the bulk-boundary correspondence, the nontrivial topological phase corresponds to the emergence of edge localized states in real space with open boundary condition. To check the topological properties in terms of the hopping modulation phase $\theta$, we exhibit the energy spectrum of the chain as a function of $\theta$ for $\phi=[-\pi / 2,0, \pi / 2]$ in Fig. 3. It can be seen that in both cases the wind number coincide with the emergence of edge states, see Fig 3 and Fig, 4 . Namely, when $\theta \in(-\pi / 2, \pi / 2)$ for $\phi \in(0,2 \pi)$, the trajectories of $\xi$ wind around the origin, the edge states appear with the ener- 

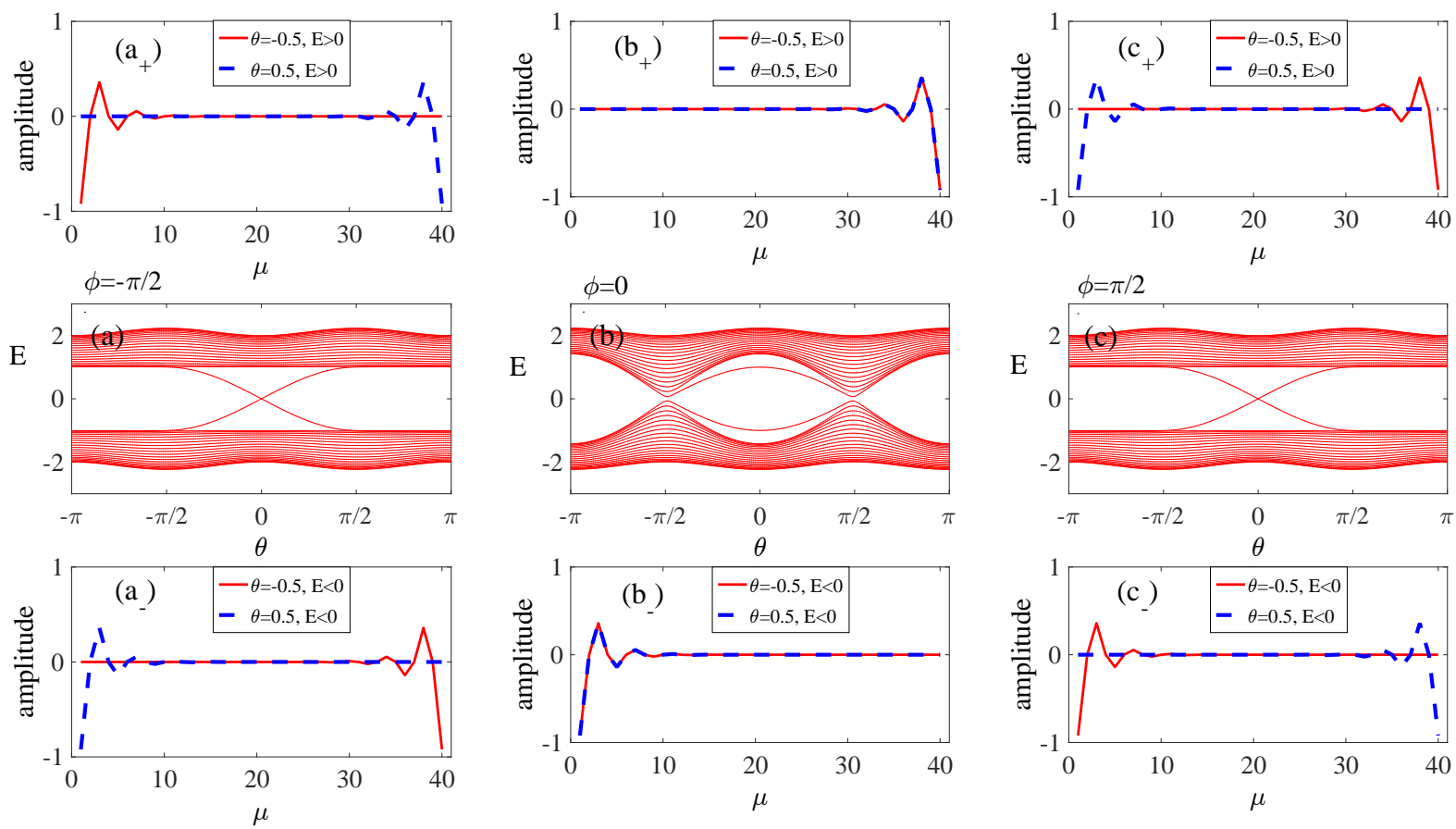

Figure 3. $\left(a_{+}\right),\left(b_{+}\right)$and $\left(c_{+}\right)$show the amplitudes of the edge states as a function of position for $\phi=-\pi / 2,0$ and $\pi / 2$ when $\theta=-0.5$ and 0.5 for the positive eigenenergies respectively. $\left(a_{-}\right),\left(b_{-}\right)$and $\left(c_{-}\right)$are those for the negative engenenergies. (a), (b) and (c) are the spectrum versus the modulation phase $\theta$ with the parameters $\phi=-\pi / 2,0$ and $\pi / 2$ respectively and the other parameters are $\lambda=0.5, v=1$ and $\mathrm{N}=40$.

gies localized in the energy gap. However in the presence of non-Hermitian loss, from the real energy spectrum, we can see that the region for the emergence of edge states shrinks. This may result from that the non-Hermitian loss 'draws' the real energy spectrum towards zero which is reflected by the deformation of the spectrum compared to those in Hermitian case in Fig. 3 . The topological region has not changed obviously according to the performance of $\xi$. And when $\theta \in(\pi / 2,3 \pi / 2)$, the origin locates outside the trajectory of $\xi$, thus edge states do not appear.

Next, we examine about the energy band and topological edge states in more details. It is easy to find that the edge states appear and their energies intersect the gap at $\theta_{c}=\pi / 2-\phi(\phi \in[0, \pi])$ and $\theta_{c}=3 \pi / 2-\phi(\phi \in[\pi, 2 \pi])$ which results to $h_{z}=0$. The degenerate point results from the particle-hole symmetry of the system, $\mathcal{S}$ : $c_{n}^{\dagger} \rightarrow(-1)^{n} c_{n}$ and $c_{n} \rightarrow(-1)^{n} c_{n}^{\dagger}$ [27. When this symmetry is broken, namely, $h_{z} \neq 0$ here, the degenerate zero energy edge state vanishes. The population of the edge states are generally localized at the ends of the chain. By checking the distribution of the wavefunctions for the edge states, we find that for the same $\theta$ or the identical energy $E$, the two edge states locate at opposite ends of the chain. And for a certain $\theta$, the two edge states locate on $\mathrm{A}$ and B-sites respectively. Since $\partial_{\theta} E$ is the group velocity of the excitation if $\theta$ is regarded as one momentum component in the $2 \mathrm{D}$ counterpart, one can see that the excitations with opposite directions of velocity locate at the opposite edges. The emergence of these boundary localized states manifests that this AAH model belongs to a nontrivial topological phase.

Compared to the Hermitian case, some intriguing properties may appear in the non-Hermitian case. Compared to the no-loss case in Fig. 3, we first calculate the energy spectrum of the chain as a function of $\theta$ with open boundary condition. The results are illustrated in Fig.4. It can be seen that not only the shape of the energy band changes but also the phase of the wavefunction reversed for the same $\theta$. However, the symmetry of the wavefunctions remain unchanged with respect to those in the Hermitian case. The edge states still appear in the energy gap in the interval $\theta \in[-\pi / 2, \pi / 2]$. The imaginary part of the eigenenergy is negative indicating decay of 

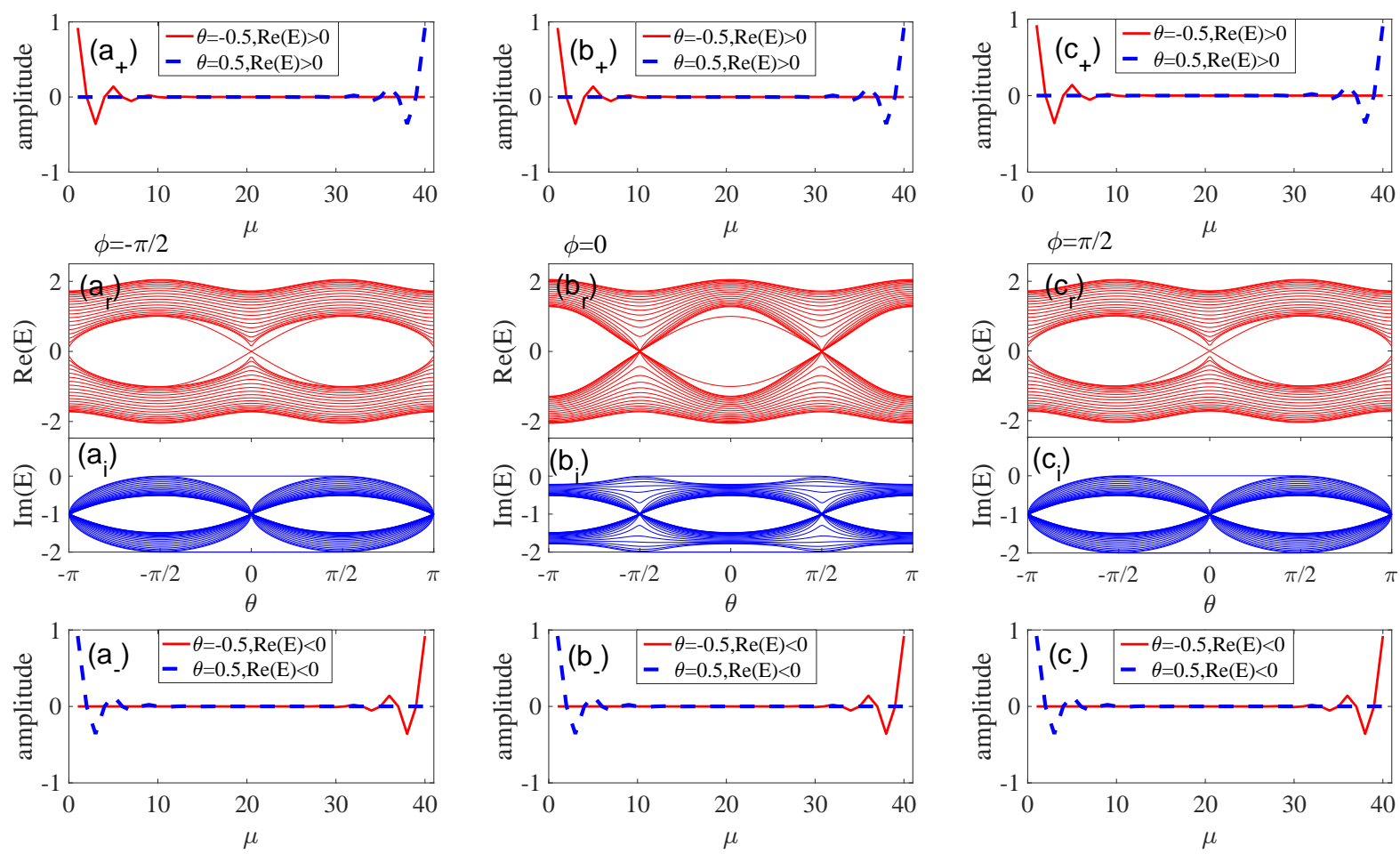

Figure 4. The real and imaginary energy spectra and distributions for the edge states same to those in Fig 3 with loss rate $\Gamma=2$ on B-sites. The other parameters are same to those in Fig 3 .
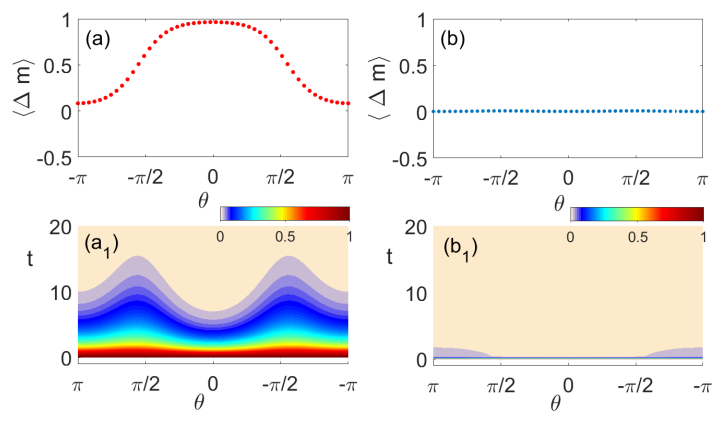

Figure 5. (a) and (b) are the average displacement versus $\theta$ in the cases of $A$-site and $B$-site initially excited respectively. $\left(a_{1}\right)$ and $\left(b_{1}\right)$ are the dynamics of the total excitation $\tau$ versus different $\theta$ s corresponding to (a) and (b) respectively. There are 20 unit cells $(\mathrm{N}=40)$ of $A-B$ combination. $\phi=0$ we have set.

the excitation except for slowly decay of the 'dark states'. Here the 'dark states' are the long-lived states that only populate on the non-loss sites which result from the interference of the wavefunction on the chain. The other witness of topology and the 'dark states' will be studied

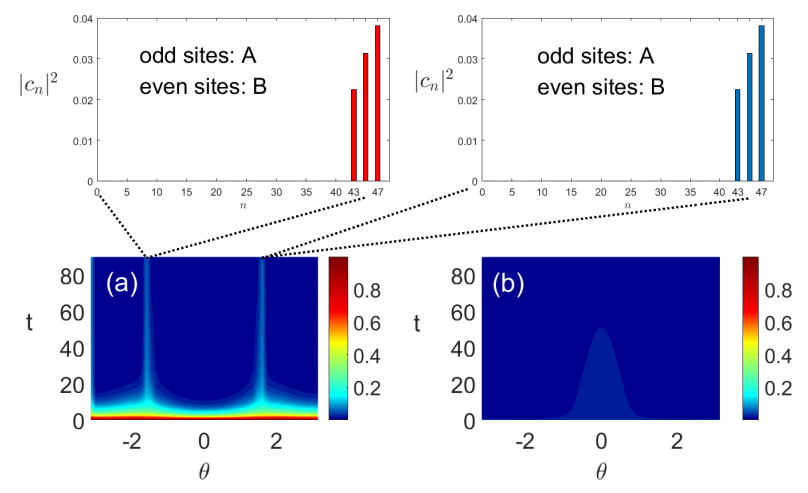

Figure 6. The evolution of the excitation $\tau$ in 9 for the initial excitation located on 1-sites (a) and 2-site (b) with the periodic boundary condition. We set $N=48$, namely there are 24 cells on the ring. The insides of the figure exhibit the distributions of the 'dark states'.

in the next section. 


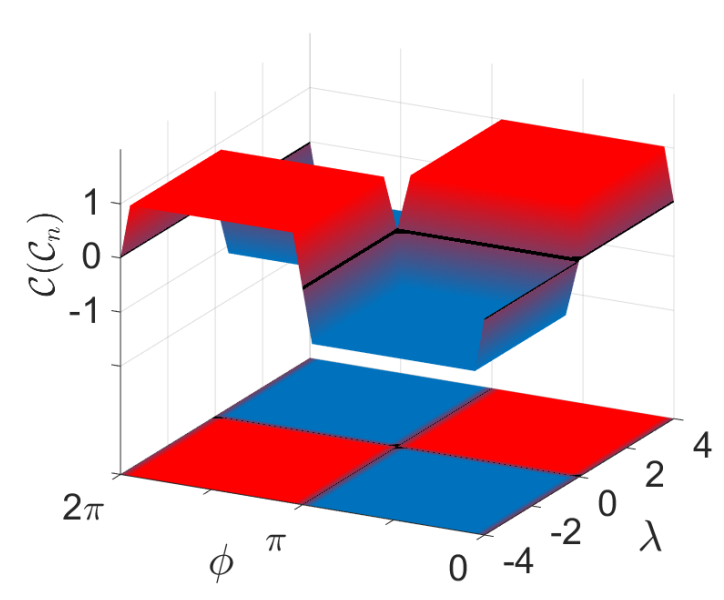

Figure 7. The Chern number versus $\phi$ and $\lambda$ in 10 and it is same for (11) versus $\phi$ and $\lambda$.

\section{A. Average displacement as a topological index}

In the non-Hermitian case, the mean displacement of the single excitation initially localized on a non-decay site can serve as a tool to witness the topological features of the system [17]. It was defined as

$$
\langle\Delta m\rangle=2 \gamma \sum_{m}\left(m-m_{0}\right) \int_{0}^{\infty} d t\left|\beta_{m}(t)\right|^{2},
$$

where $\beta_{m}$ is the amplitude of the wavefunction on site $B$ in unit cell $m$ at time $t$ and $m_{0}$ denotes the position for the initial single excitation. For multi-excitation case, further work for $\langle\Delta m\rangle$ may be investigated in the future. Transforming it to momentum space by Fourier transformation, the average displacement equals to a wind number of the relative phase between two components of the Bloch wavefunction [17] and provides a feasible methodology to unraveling the topological properties of the system rather than probing the edge states [28, 30].

It can be seen that $\langle\Delta m\rangle$ depends on the dynamical history of the wavefunction. The dynamics for the wavefunction is governed by the equations:

$$
\begin{aligned}
\dot{\alpha_{n}}= & -i(1-\lambda \cos (\theta)) \beta_{n}-i(1+\lambda \cos (\theta)) \beta_{n-1} \\
& +i v \cos \left(\theta_{v}\right) \alpha_{n}, \\
\dot{\beta_{n}}= & -i(1-\lambda \cos (\theta)) \alpha_{n}-i(1+\lambda \cos (\theta)) \alpha_{n+1} \\
& -i v \cos \left(\theta_{v}\right) \beta_{n}-2 \Gamma \beta_{n},
\end{aligned}
$$

where $\alpha_{n}$ and $\beta_{n}$ are the amplitudes for the wavefunction on $A$ and $B$ sites respectively. The loss effect resulting from the minus complex on-site potentials on B-sites can be seen from these dynamical equations. By integrating the dynamical equations, we gain the average displacement numerically.

We plot $\langle\Delta m\rangle$ versus $\theta$ in Fig. 5 (a) and (b). Compared to the energy spectrum in Fig. 3, we can see that $\theta= \pm \pi / 2$ are the transition points for $\langle\Delta m\rangle$ which coincide with the appearance of edge state versus $\theta$. And the total excitation which is defined as

$$
\tau(t)=\sum_{m}\left|\alpha_{m}(t)\right|^{2}+\left|\beta_{m}(t)\right|^{2}
$$

would be longer at the transition points. Then we exhibit the dynamics of the total excitation $\tau$ versus $\theta$ in Fig. 5 $\left(a_{1}\right)$ and $\left(b_{1}\right)$. It can be seen that while $\theta= \pm \pi / 2$, the lifetime of the excitation is prolonged obviously. This results from the existence of 'dark state'. To exhibit the 'dark states', the distribution of the wavefunction with periodic boundary condition is checked in Fig.6. It can be seen that there are long-lived states with excitation locating at the non-loss sites, namely, the 'dark state'. Such states result from the coherence of the wavefunction on the chain when $\theta= \pm \pi / 2$. That means with the periodic boundary condition, even there is the loss on B-sites, the dark state can survive in a long time scale. However, the coupling between $\mathrm{A}$ and $\mathrm{B}$ sites leads to damping of the excitation gradually.

\section{TOPOLOGICAL PROPERTIES IN TERMS OF ON-SITE MODULATION PHASE}

In this section, we study the effect of loss on the topological properties in terms of phase $\phi$ in the on-site modulation. If we assume $\theta_{v}=\theta+\phi$, the system exhibits intriguing topological properties in terms of $\phi$. By transforming the system to momentum space, the topological properties can be quantified by Chern number defined by

$$
\mathcal{C}(\phi)=\frac{1}{4 \pi} \iint_{B Z} d k d \theta\left(\partial_{k} \vec{h} \times \partial_{\theta} \vec{h}\right),
$$

where $\vec{h}=\left(h_{x}, h_{y}, h_{z}\right)$. From the other point of view, the Chern number can be calculated via the $n$th energy eigenstate, it reads

$$
\mathcal{C}_{n}(\phi)=-\frac{1}{2 \pi} \iint_{B Z} d k d \theta\left(\partial_{k} A_{\theta}^{(n)} \times \partial_{\theta} A_{k}^{(n)}\right),
$$

here $A_{j}^{(n)}(j=k, \theta)$ are the Berry connection given by $A_{j}^{n}=-i\left\langle u^{(n)}\left|\partial_{j}\right| u^{n}\right\rangle$. These two Chern numbers are identical to describe the topological properties of the system. Later, we will take the Chern number in 10 as the topological index.

In Fig.??, we show the Chern number $\mathcal{C}\left(\mathcal{C}_{n}\right)=$ $\operatorname{sign}(\lambda \sin (\phi))$ as a function of $\phi$ and $\lambda$. With $\lambda=0.5$, we present the topological characters and the energy spectra versus $\phi$ in Fig . 8. It can be seen that $\phi=\pi$ is the critical point for topological phase transition where the energy gap reopens after closing in the Brillouin zone $\phi \in[0,2 \pi]$. The origin is wrapped by the torus of $h_{x}-h_{y}-h_{z}$ in the 


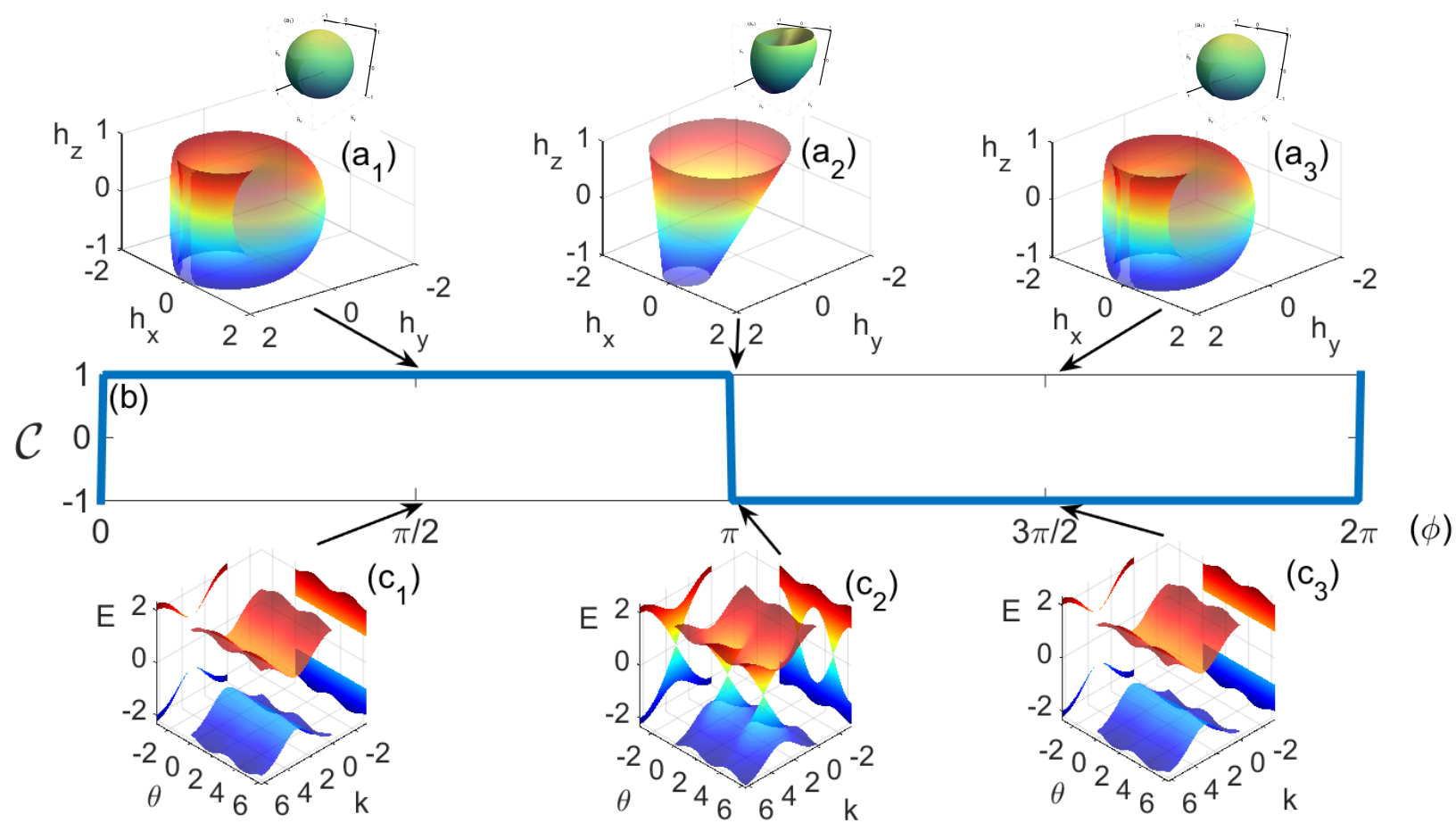

Figure 8. $\left(a_{1}\right),\left(a_{2}\right)$ and $\left(a_{3}\right)$ are the graphical representations of the Chern number in the space of $h_{x}-h_{y}-h_{z}$ for $\phi=\pi / 2, \pi$ and $3 \pi / 2$ respectively. The insets are the normalized graphical representations for the Chern number. $\left(c_{1}\right),\left(c_{2}\right)$ and $\left(c_{3}\right)$ are the energy spectra corresponding to $\left(a_{1}\right),\left(a_{2}\right)$ and $\left(a_{3}\right)$ respectively. $(b)$ exhibits the Chern number defined in 10 . We have set $\lambda=0.5$ and $v=1$.

Brillouin $\kappa, \theta \in[0,2 \pi]$ when $\phi \neq \pi$ where $n=0,1$. When $\phi=\pi$, the torus of $h_{x}-h_{y}-h_{z}$ degenerate to a closed ribbon across the origin. The insets of $\left(a_{1}\right),\left(a_{2}\right)$ and $\left(a_{3}\right)$ in Fig 8 show $\bar{h}_{i}, i=x, y, z$ normalized by $|\bar{h}|$. When the Chern number is nonvanishing, the sphere of $\bar{h}$ wraps the origin. And correspondingly, the edge states appear in open boundary condition in real space. We exhibit the edge spectrum as a function of $\phi$ in the Supplement material by a movie. We can see in the movie that the edge state appears except $\phi=0$ or $\pi$ in the Brillouin zone with $\Gamma=0$. Considering the energy band in momentum space, it can be seen that when $\phi=\pi$, two Dirac points appear. Since the linear dispersion relation near the cones, the excitations with positive and negative energies act like massless particles.

Next, we examine the influence of the non-Hermitian loss to the Chern number $\mathcal{C}$, the results are shown in Fig 4. It can be seen that with the increasing of the loss strength $\Gamma$, the nontrivial topological region is shrunk. Correspondingly, the touching points in the real energy spectrum become lines in the momentum space. In Fig[10, we exhibit the influence of the loss to the real energy spectrum. The length of the line of $\operatorname{Re}(E)=0$ corresponds to the shrink of the region for the emergence of edge states with the open energy gap.

We show the shrink of the region for the emergence of edge states by four different $\Gamma$ s in the movie in the Supplement material. We can see that with increasing of $\Gamma$, the interval of $\phi$ for the band closing points increase. But the edge states do not disappear. Then we conclude that the non-Hermitian loss 'draws' the energy spectrum towards zero but dissolve the edge states. The nonvanishing Chern number shown in Fig. 9 corresponds to the existence of edge state when the energy gap open.

In the non-Hermitian case, the degenerate points (often called exceptional points) have interesting properties [31. These points are different from the degenerate points in Hermitian case since the eigenstates at these points usually coalesce into one self-orthogonal state. In the non-Hermitian case, the dispersion relation is $E= \pm(\mathcal{R}+i \mathcal{I})$ where $\mathcal{R}=\sqrt{\frac{1}{2}(F+G)}$ and $\mathcal{I}=\operatorname{sign}(-v \cos (\theta+\phi)) \sqrt{\frac{1}{2}(F-G)} . F=\sqrt{G^{2}+4 B^{4} \Gamma^{2}}$ 


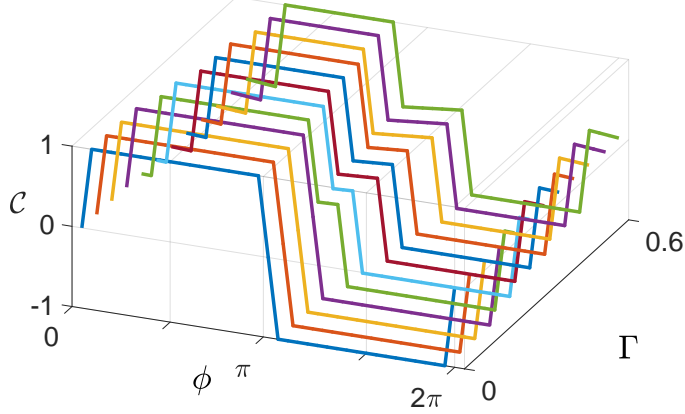

Figure 9. The Chern number versus $\phi$ for a range of nonHermitian loss strength $\Gamma$ are shown. The other parameters are same to those in Fig. 8.

and $G=A^{2}+B^{2}+C-\Gamma^{2}$. We consider the case of $\theta_{v}=$ $\pi / 2$, the energy spectrum $E= \pm \sqrt{G}$. The exceptional points in this case fulfill the condition: $4 \cos ^{2}(k / 2)+$ $4 \lambda^{2} \cos ^{2}(\theta) \sin ^{2}(k / 2)=\Gamma^{2}$ in the $k-\theta$ space. We plot the energy spectrum and the exceptional points in Fig 11 . We find that the exceptional points constitute two loops in the parameter space of $k-\theta$. There are intriguing properties of the exceptional points, e.g., $(k, \theta)=(\pi, \pi)$ is one exceptional point with the self-orthogonal eigenvector $e^{i \mu}\left[\begin{array}{ll}i & 1\end{array}\right]^{T}$ corresponding to the eigenenergy 0 where $\mu$ is an gauge factor.
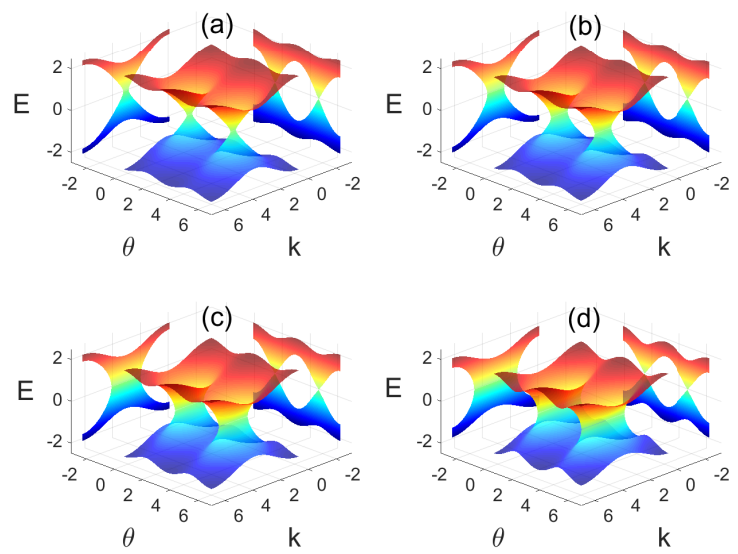

Figure 10. (a), (b), (c) and (d) are the real energy spectrum in momentum space for $\Gamma=0.1,0.3,0.5$ and 0.7 respectively. $\phi=\pi$ here. The other parameters are the same as those in Fig. 8

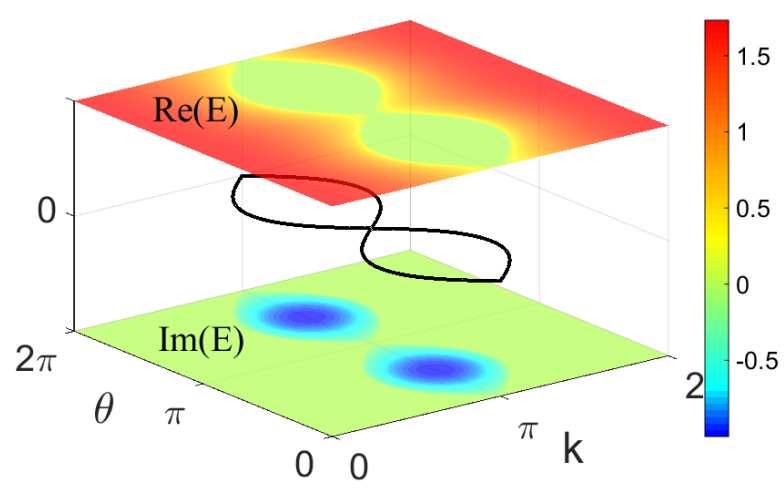

Figure 11. Energy spectrum of the non-Hermitian Hamiltonian and the exceptional points in momentum space. The black loops consists of the exceptional points when $\theta_{v}=\pi / 2$.

\section{ROBUSTNESS OF THE ZERO ENERGY EDGE STATE}

The existence of the edge states is a nontrivial topological signature in this model. Among the edge states, the zero energy degenerate states are very interesting. In the following by numerical simulations we study the robustness of the zero energy edge state against four kinds of disorders. The results are shown in Fig 12 . From the numerical simulations, we can see that although the details of the robustness of the zero mode against the disorders are different, the states are robust against these disorders but $\delta v$. The zero energy edge state is fragile under the effect of disorders in $\delta v$ due to the broken particle-hole symmetry as discussed in section II Regardless of the non-zero eigenenergy, the degenerate states are localized in the band gap. With the increasing of $\delta \Gamma$, the eigenenergies tend to zero. In the previous discussions of this work, we found that with the increasing of the loss, the region for the closing of the bulk band increases. Thus it maybe conclude that the loss can drive the real energy band towards zero. So the $E=0$ degenerate eigenvalue is robust against disorders as long as the particle-hole symmetry is preserved. The disorders of on-site potentials immediately destroy this symmetry which lead the zero energy edge state split into non-zero states.

\section{EXPERIMENTAL SETUP}

A setup to observe the predicted topological properties can be realized in coupled single mode optical waveguides [7, 16, 18, 32. Each waveguide represents a site and the time evolution is equivalent to the light excitation propagating in the waveguides. The lattice can be fabricated in silica glass by femtosecond direct laser writing technique 33 or by high resolution large field 

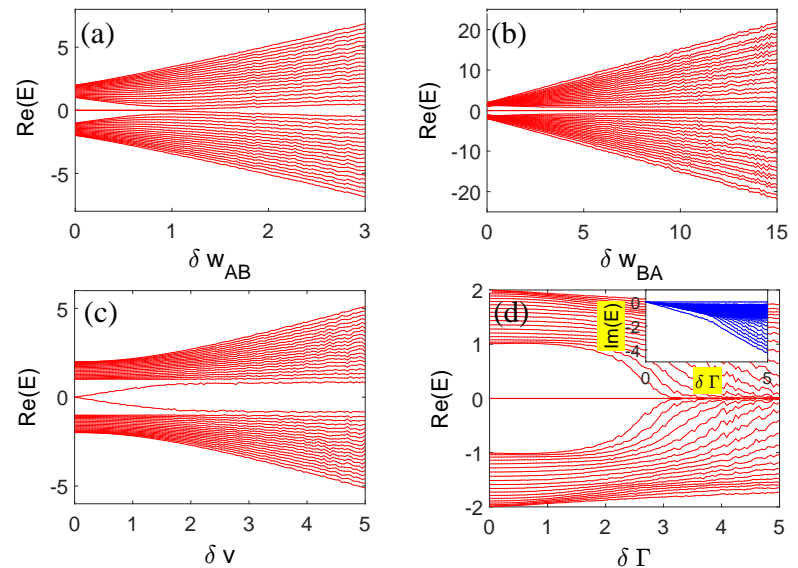

Figure 12. In (a), (b), (c) and (d): $\delta w_{A B}$ and $\delta w_{B A}$ are the disorders for the intra-cell and inter-cell hoppings, respectively, and $\delta v$ and $\delta \Gamma$ are those for the on-site potentials and loss. In each numerical simulation, we assume that the disorders are randomly distributed on the chain with strengths uniformly distribute in $[0, \delta x]$ ( $\delta \Gamma$ locate on B-sites only), here $\delta x$ are $\delta w_{A B}, \delta w_{B A}, \delta v$ and $\delta \Gamma$. Here we have set $\theta=0$ and $\phi=\pi / 2$ when the zero energy edge state exists. The (non-Hermitian) loss is added to the Hermitian Hamiltonian on B-sites. Each line is an average over 100 simulations.

e-beam lithography technique on AlGaAs substrate [16]. The hopping modulation can be tuned by varying the spacing between the waveguides which provides a way to determine $\lambda$ and $\theta$. The on-site potentials can be modulated by changing the widths of the waveguides which determine $v$ and $\phi$. The loss is introduced by bending the even-waveguides wiggly perpendicular to the plane of the silica glass [18 in the trigonometrical manner along the propagating direction of the light or by varying the etch depth of the waveguides on AlGaAs substrate. Since the intrinsic loss of the waveguide is identical for all sites, it can be factored out. And the fluorescence microscopy technique can be employed to observe the light intensity propagating along the waveguides to check the topological properties. Fig 1 shows a sketch for such a setup.

\section{CONCLUSION}

In this work, we have studied the influence of loss to the topological properties of an extended AAH model in terms of hopping and on-site modulation phases. We found that the parameter region for the emergence of the edge localized states with open band gap is shrunk in the presence of loss. We also examine the average displacement of the single excitation and find that it can witness the topological properties of the system. Long-lived 'dark states' for the chain are shown in the periodic boundary condition. In terms of on-site modulation phase, we found that compared to the Hermitian case, the region for the nontrivial topological phase is also shrunk in the presence of loss. And the zero-energy edge states are robust against intra-cell, inter-cell and non-Hermitian loss disorders but fragile against those in the on-site potentials since the particle-hole symmetry is broken in the last case. The energy spectrum tends towards zero when the loss disorders increase. Finally, we propose an experimental setup based on coupled waveguides to implement this model.

\section{ACKNOWLEDGMENTS}

We thank Prof. L. C. Wang at Dalian University of Technology for valuable discussions. This work is supported by the National Natural Science Foundation of China (Grant No. 11534002 and 61475033).
[1] S. Aubry and G. Andre, Analyticity breaking and Anderson localization in incommensurate lattices, Ann. Isr. Phys. Soc. 3, 133 (1980).

[2] P.G. Harper, The General Motion of Conduction Electrons in a Uniform Magnetic Field, with Application to the Diamagnetism of Metals, Proc. Phys. Soc. London Sect. A 68, 874 (1955).

[3] Douglas R. Hofstadter, Energy levels and wavefunctions of Bloch electrons in rational and irrational magnetic fields, Phys. Rev. B 14, 2239 (1976).

[4] M. Aidelsburger, M. Atala, M. Lohse, J. T. Barreiro, B. Paredes, and I. Bloch, Realization of the Hofstadter Hamiltonian with Ultracold Atoms in Optical Lattices, Phys. Rev. Lett. 111, 185301 (2013).

[5] Hirokazu Miyake, Georgios A. Siviloglou, Colin J. Kennedy, William Cody Burton, and Wolfgang Ketterle, Realizing the Harper Hamiltonian with Laser-Assisted Tunneling in Optical Lattices, Phys. Rev. Lett. 111,
$185302(2013)$.

[6] Li-Jun Lang, Xiaoming Cai, and Shu Chen, Edge States and Topological Phases in One-Dimensional Optical Superlattices, Phys. Rev. Lett. 108, 220401 (2012).

[7] Yaacov E. Kraus, Yoav Lahini, Zohar Ringel, Mor Verbin, and Oded Zilberberg, Topological States and Adiabatic Pumping in Quasicrystals, Phys. Rev. Lett. 109, 106402 (2012).

[8] Yaacov E. Kraus and Oded Zilberberg, Topological Equivalence between the Fibonacci Quasicrystal and the Harper Model, Phys. Rev. Lett. 109, 116404 (2012).

[9] Feng Mei, Shi-Liang Zhu, Zhi-Ming Zhang, C. H. Oh, and N. Goldman, Simulating $Z_{2}$ topological insulators with cold atoms in a one-dimensional optical lattice, Phys. Rev. A 85, 013638 (2012).

[10] Feng Mei, Jia-Bin You, Dan-Wei Zhang, X. C. Yang, R. Fazio, Shi-Liang Zhu, and L. C. Kwek, Topological insulator and particle pumping in a one-dimensional shaken 
optical lattice, Phys. Rev. A 90, 063638 (2014).

[11] R. B. Laughlin, Quantized Hall conductivity in two dimensions, Phys. Rev. B 23, 5632(R) (1981).

[12] D. J. Thouless, M. Kohmoto, M. P. Nightingale, and M. den Nijs, Quantized Hall Conductance in a TwoDimensional Periodic Potential, Phys. Rev. Lett. 49, 405 (1982).

[13] Yasuhiro Hatsugai, Chern number and edge states in the integer quantum Hall effect, Phys. Rev. Lett. 71, 3697 (1993).

[14] Y. Avron, R. Seiler, and B. Shapiro, Generic properties of quantum Hall Hamiltonians for finite systems, Nucl. Phys. B 265, 364 (1986).

[15] M. Z. Hasan and C. L. Kane, Colloquium: Topological insulators, Rev. Mod. Phys. 82, 3045 (2010).

[16] Y. Lahini, R. Pugatch, F. Pozzi, M. Sorel, R. Morandotti, N. Davidson, and Y. Silberberg, Observation of a Localization Transition in Quasiperiodic Photonic Lattices, Phys. Rev. Lett. 103, 013901 (2009).

[17] M. S. Rudner and L. S. Levitov, Topological Transition in a Non-Hermitian Quantum Walk, Phys. Rev. Lett. 102, 065703 (2009).

[18] Julia M. Zeuner, Mikael C. Rechtsman, Yonatan Plotnik, Yaakov Lumer, Stefan Nolte, Mark S. Rudner, Mordechai Segev, and Alexander Szameit, Observation of a Topological Transition in the Bulk of a Non-Hermitian System, Phys. Rev. Lett. 115, 040402 (2015).

[19] S. Das Sarma, S. He, and X.C. Xie, Mobility Edge in a Model One-Dimensional Potential, Phys. Rev. Lett. 61, 2144 (1988).

[20] D.J. Thouless, Localization by a Potential with Slowly Varying Period, Phys. Rev. Lett. 61, 2141 (1988).

[21] S. Das Sarma, S. He, and X. C. Xie, Localization, mobility edges, and metal-insulator transition in a class of one-dimensional slowly varying deterministic potentials, Phys. Rev. B 41, 5544 (1990).

[22] J. Biddle, B. Wang, D.J. Priour, and S. Das Sarma, Localization in one-dimensional incommensurate lattices beyond the Aubry-Andr model, Phys. Rev. A 80, 021603
(2009).

[23] J. Biddle and S. Das Sarma, Predicted Mobility Edges in One-Dimensional Incommensurate Optical Lattices: An Exactly Solvable Model of Anderson Localization, Phys. Rev. Lett. 104, 070601 (2010).

[24] J.H. Han, D.J. Thouless, H. Hiramoto, and M. Kohmoto, Critical and bicritical properties of Harpers equation with next-nearest-neighbor coupling, Phys. Rev. B 50, 11365 (1994).

[25] M. Kohmoto, Metal-Insulator Transition and Scaling for Incommensurate Systems, Phys. Rev. Lett. 51, 1198 (1983).

[26] H. Hiramoto and M. Kohmoto, Scaling analysis of quasiperiodic systems: Generalized Harper model, Phys. Rev. B 40, 8225 (1989).

[27] Shinsei Ryu and Yasuhiro Hatsugai, Topological Origin of Zero-Energy Edge States in Particle-Hole Symmetric Systems, Phys. Rev. Lett. 89, 077002 (2002).

[28] B. Andrei Bernevig, Taylor L. Hughes, Shou-Cheng Zhang, Quantum Spin Hall Effect and Topological Phase Transition in HgTe Quantum Wells, Science 314,1757 (2006).

[29] Mikael C. Rechtsman, Julia M. Zeuner, Yonatan Plotnik, Yaakov Lumer, Daniel Podolsky, Felix Dreisow, Stefan Nolte, Mordechai Segev and Alexander Szameit, Photonic Floquet topological insulators, Nature 496, 196 (2013).

[30] D. Hsieh, D. Qian, L. Wray, Y. Xia, Y. S. Hor, R. J. Cava and M. Z. Hasan1, A topological Dirac insulator in a quantum spin Hall phase, Nature 452, 970 (2008).

[31] W. D. Heiss, The physics of exceptional points, J. Phys. A 45,444016 (2012).

[32] Mohammad Hafezi, Eugene A. Demler, Mikhail D. Lukin and Jacob M. Taylor, Robust optical delay lines with topological protection, Nature Physics 7, 907 (2011).

[33] Alexander Szameit and Stefan Nolte, Discrete optics in femtosecond-laser-written photonic structures, Journal of Physics B: Atomic, Molecular and Optical Physics, 43, 163001 (2010). 